\title{
Article
}

\section{Linear Bundle of Lie Algebras Applied to the Classification of Real Lie Algebras}

\author{
Alina Dobrogowska ${ }^{*,+}+\mathbb{D}$ and Karolina Wojciechowicz ${ }^{\dagger}$ \\ Faculty of Mathematics, University of Bialystok, Ciołkowskiego 1M, 15-245 Białystok, Poland; \\ kwojciechowicz@math.uwb.edu.pl \\ * Correspondence: alina.dobrogowska@uwb.edu.pl \\ + These authors contributed equally to this work.
}

check for updates

Citation: Dobrogowska, A.; Wojciechowicz, K. Linear Bundle of Lie Algebras Applied to the Classification of Real Lie Algebras. Symmetry 2021, 13, 1455. https:// doi.org/10.3390/sym13081455

Academic Editor: José Carlos R. Alcantud

Received: 26 June 2021

Accepted: 5 August 2021

Published: 9 August 2021

Publisher's Note: MDPI stays neutral with regard to jurisdictional claims in published maps and institutional affiliations.

Copyright: (c) 2021 by the authors. Licensee MDPI, Basel, Switzerland. This article is an open access article distributed under the terms and conditions of the Creative Commons Attribution (CC BY) license (https:// creativecommons.org/licenses/by/ $4.0 /)$.
Abstract: We present a new look at the classification of real low-dimensional Lie algebras based on the notion of a linear bundle of Lie algebras. Belonging to a suitable family of Lie bundles entails the compatibility of the Lie-Poisson structures with the dual spaces of those algebras. This gives compatibility of bi-Hamiltonian structure on the space of upper triangular matrices and with a bundle at the algebra level. We will show that all three-dimensional Lie algebras belong to two of these families and four-dimensional Lie algebras can be divided in three of these families.

Keywords: Lie algebra; linear bundle of Lie algebras; quasisimple orthogonal algebra; Poisson manifold; bi-Hamiltonian structure

\section{Introduction}

To begin, we recall the definition of a Lie bundle. Let $V, W$ be finite dimensional vector spaces. If for any $X, Y \in V$ and any $S \in W$, we can define Lie bracket on $V$

$$
(X, Y) \mapsto[X, Y]_{S}
$$

which is linear in $S$, then the pair $(V, W)$ is called a linear bundle of Lie algebras or a Lie bundle, see [1-3]. A simple consequence of the definition is that the Lie brackets are compatible in the sense that their linear combination is again a Lie bracket

$$
[\cdot, \cdot]_{\alpha S+\beta T}=\alpha[\cdot, \cdot]_{S}+\beta[\cdot, \cdot]_{T}
$$

where $S, T \in W, \alpha, \beta \in \mathbb{R}$.

A classical example of such a structure is obtained by taking the space of skewsymmetric matrices $n \times n$ as $V$, which we denote by $\mathcal{A}(n)$, and the space of symmetric matrices as $W$, which we denote by $\mathcal{S}(n)$. A family of compatible brackets for this case is given by the following formula

$$
[X, Y]_{S}=X S Y-Y S X
$$

where $X, Y \in \mathcal{A}(n), S \in \mathcal{S}(n)$. As a special case for $S=1$, we obtain the standard matrix commutator and Lie algebra $\mathfrak{s o}(n)$. The Lie bundle $(\mathcal{A}(n), \mathcal{S}(n))$ appears in many places in the theory of integrable systems. Morosi and Pizzocchero, for example, in [4], used those brackets to construct recursion giving the Mischenko and Manakov integrals of motion for the Euler equation of an $n$-dimensional rigid body. In the paper [1], Bolsinov and Borisov applied those brackets to investigate the problem of multidimensional Euler and Clebsh equations. Those brackets were also used to analyze Clebsh and Neumann systems in [3]. We recommend the articles [5-7], where the bracket (2) or some of its modification were used to consider some integrable systems. 
The Lie bundle $(\mathcal{A}(n), \mathcal{S}(n))$ also suggests another linear bundle of Lie algebras $(\mathcal{S}(n), \mathcal{A}(n))$ with family of brackets given by

$$
[S, T]_{X}=S X T-T X S,
$$

where $T, S \in \mathcal{S}(n), X \in \mathcal{A}(n)$. This structure, for example, was considered in [8].

Brackets of this type were already described by Cantor and Persits in [9]. A detailed analysis of the situation for $M_{m \times n}(\mathbb{R})$ was done by Dali in [10]. In addition, Kiranagi with collaborators studied Lie algebra bundles in a general situation, see, e.g., [11].

If we have a Lie algebra $(\mathfrak{g},[\cdot, \cdot])$, then we can think about its generalization in two different ways. On one hand, we can deform the Lie bracket $[\cdot, \cdot]$, see, for example [12], without changing the set $\mathfrak{g}$. On the other hand, we can leave the Lie bracket in the unchanged form and only deform the set by introducing some parameters, which will give us new algebra if we let some of those parameters tend to zero, see $[13,14]$. In our article, we will consider both of these approaches-contractions and deformations of Lie algebras.

Our base Lie algebra is $\mathfrak{s o}(n)$. We already know that the modification of the Lie bracket by a symmetric matrix (2) gives us again a Lie algebra. We can expand the set of skewsymmetric matrices by introducing $n-1$ real parameters $a_{1}, \ldots, a_{n-1}$, see $[15,16]$. This construction gives us a large family of algebras, which are called quasisimple Cayley-Klein algebras of the orthogonal type. Analysis of this kind of algebras applied to bi-Hamiltonian structures, which they generate, was given in [6,17]. Articles [18,19] show us those algebras in consideration with Minkowski spacetime.

Moreover, we will also modify the set of symmetric matrices, for instance, by introducing parameters $a_{1}, \ldots, a_{n-1}$. After that modification, with some given parameters, we will still have a Lie bundle. We will analyze when (for different parameter systems) those Lie bundles will be compatible, in the sense that their linear combination will give us a different Lie bundle, which corresponds to the compatible Poisson structure on the dual space (the same for all of these algebras). Next, we will show that the notion of bi-Hamiltonian structure, which is very useful for studying integrable systems, see [20], can also be used in the classification of Lie algebras. The standard classification of Lie algebras is based on the Levi-Malcev theorem, which says that any finite-dimensional Lie algebra can be represented as the semi-direct sum of the radical $\tau$ and a semisimple subalgebra $\mathfrak{a}$, i.e., $\mathfrak{g}=\mathfrak{a} \ltimes \tau$. Then, one needs a classification of all semisimple algebras, all solvable algebras and all possible linear actions of the semisimple algebras $\mathfrak{a}$ on the radical $\tau$. The problem of classifying real Lie algebras is completely solved for Lie algebras up to dimension six. Bianki [21] first classified the tree-dimensional real Lie algebras. All the possible real Lie algebras of a dimension up to and including four were listed by Mubarakzyanov [22]. For more detail, see [23-27] and the references therein. In our considerations, we will look at this problem from another point of view and attempt the classification according to membership of suitable Lie bundles.

The paper is organized as follows. In the beginning, we will recall basic facts about Poisson brackets (in particular, linear structure) and bi-Hamiltonian structure. Next, we discuss generalizations of the Lie bundle $(\mathcal{A}(n), \mathcal{S}(n))$ by the introduction of real parameters $a_{1}, \ldots, a_{n-1}$ and partially replacing the vector space indexing Lie brackets (symmetric matrices or quasisimple symmetric matrices) by any matrices. Section 3 is devoted to analysis of families of brackets associated with real three-dimensional Lie algebras. In the next section, we consider Lie bundles generating real four-dimensional Lie algebras.

\section{Bi-Hamiltonian Structure and Lie Bundles Parametrized by a Finite Sequence of Parameters}

The main aim of this section is to recall basic facts about Lie algebras, Poisson manifolds and the relation between those two. 
Let us consider a finite-dimensional manifold $M$. A Poisson bracket is a bilinear and skew-symmetric operation that satisfies the following conditions:

$$
\{\{f, g\}, h\}+\{\{g, h\}, f\}+\{\{h, f\}, g\}=0, \quad\{f, g h\}=g\{f, h\}+\{f, g\} h,
$$

for all $f, g, h \in C^{\infty}(M)$. The first of these conditions is usually called the Jacobi identity, and the second is the Leibniz rule. A manifold $M$ with this bracket is called a Poisson manifold $(M,\{\cdot, \cdot\})$.

If $\mathbf{x}=\left(x_{1}, \ldots, x_{n}\right)$ is a system of local coordinates on $M$, then the Poisson bracket can be presented in the form

$$
\{f, g\}(\mathbf{x})=\sum_{i, j=1}^{n} \pi_{i j}(\mathbf{x}) \frac{\partial f}{\partial x_{i}} \frac{\partial g}{\partial x_{j}},
$$

where $\pi \in \Gamma\left(\wedge^{2} T M\right)$ is called a Poisson tensor. The components of this tensor are given by the formula $\pi_{i j}(\mathbf{x})=-\pi_{j i}(\mathbf{x})=\left\{x_{i}, x_{j}\right\}$, and it satisfies the following system of equations, equivalent to the Jacobi identity,

$$
\sum_{s=1}^{n}\left(\frac{\partial \pi_{i j}}{\partial x_{s}} \pi_{s k}+\frac{\partial \pi_{k i}}{\partial x_{s}} \pi_{s j}+\frac{\partial \pi_{j k}}{\partial x_{s}} \pi_{s i}\right)=0,
$$

which is equivalent to the vanishing of the Schouten-Nijenhuis bracket.

If $\pi$ depends on $\mathbf{x}$ in a linear way, we say we have a linear Poisson structure on $M$.

In this paper, an important example of Poisson structure will be the so-called LiePoisson bracket. Let $\mathfrak{g}$ be a real $n$-dimensional Lie algebra. It is a well-known fact that on the dual space $\mathfrak{g}^{*}$, there exists a canonical Poisson structure. This bracket is called Lie-Poisson, and it is given by the following formula

$$
\{f, g\}(\mathbf{x})=\langle\mathbf{x},[d f(\mathbf{x}), d g(\mathbf{x})]\rangle,
$$

where $d f(\mathbf{x}), d g(\mathbf{x}) \in\left(\mathfrak{g}^{*}\right)^{*} \cong \mathfrak{g}$. Let $\left\{e_{1}, \ldots, e_{n}\right\}$ be a basis of $\mathfrak{g}$. Using the structure constants $c_{i j}^{k}$ of this Lie algebra $\left[e_{i}, e_{j}\right]=\sum_{k=1}^{n} c_{i j}^{k} e_{k}$, one can express the Poisson tensor as a linear function $\left\{x_{i}, x_{j}\right\}=\sum_{k=1}^{n} c_{i j}^{k} x_{k}$. This gives a one-to-one correspondence between the linear Poisson structures and Lie algebras.

We say that two Poisson tensors $\pi_{1}$ and $\pi_{2}$ are compatible if any linear combination

$$
\pi=\alpha \pi_{1}+\beta \pi_{2}, \quad \alpha, \beta \in \mathbb{R},
$$

is also a Poisson tensor. The Poisson structures $\pi_{1}$ and $\pi_{2}$ on $M$ are compatible if and only if their Schouten-Nijenhuis bracket vanishes, which means that

$$
\sum_{s=1}^{n}\left(\pi_{2, s k} \frac{\partial \pi_{1, i j}}{\partial x_{s}}+\pi_{1, s k} \frac{\partial \pi_{2, i j}}{\partial x_{s}}+\pi_{2, s j} \frac{\partial \pi_{1, k i}}{\partial x_{s}}+\pi_{1, s j} \frac{\partial \pi_{2, k i}}{\partial x_{s}}+\pi_{2, s i} \frac{\partial \pi_{1, j k}}{\partial x_{s}}+\pi_{1, s i} \frac{\partial \pi_{2, j k}}{\partial x_{s}}\right)=0 .
$$

The manifold $M$ equipped with two compatible Poisson structures $\pi_{1}$ and $\pi_{2}$ is called the bi-Hamiltonian manifold, and we denote it as $\left(M, \pi_{1}, \pi_{2}\right)$.

Let us consider sets of square matrices

$$
\mathcal{A}_{a_{1}, \ldots, a_{n-1}}(n)=\left\{X=\left(x_{i j}\right) \in M_{n}(\mathbb{R}): x_{i j}=-a_{i} \ldots a_{j-1} x_{j i} \quad \text { for } \quad j>i, \quad x_{i i}=0\right\}
$$

and

$$
\mathcal{S}_{a_{1}, \ldots, a_{n-1}}(n)=\left\{S=\left(s_{i j}\right) \in M_{n}(\mathbb{R}): s_{i j}=a_{i} \ldots a_{j-1} s_{j i} \quad \text { for } \quad j>i\right\},
$$


where $a_{i}, i=1, \ldots, n-1$, are fixed real numbers. Elements of these sets can be represented as follows

$$
\begin{gathered}
X=\left(\begin{array}{ccccc}
0 & -a_{1} x_{21} & -a_{1} a_{2} x_{31} & \ldots & -a_{1} a_{2} \ldots a_{n-1} x_{n 1} \\
x_{21} & 0 & -a_{2} x_{32} & \ldots & -a_{2} a_{3} \ldots a_{n-1} x_{n 2} \\
x_{31} & x_{32} & 0 & \ldots & -a_{3} a_{4} \ldots a_{n-1} x_{n 3} \\
\vdots & \vdots & \vdots & \ddots & \vdots \\
x_{n 1} & x_{n 2} & x_{n 3} & \ldots & 0
\end{array}\right), \\
S=\left(\begin{array}{ccccc}
s_{11} & a_{1} s_{21} & a_{1} a_{2} s_{31} & \ldots & a_{1} a_{2} \ldots a_{n-1} s_{n 1} \\
s_{21} & s_{22} & a_{2} s_{32} & \ldots & a_{2} a_{3} \ldots a_{n-1} s_{n 2} \\
s_{31} & s_{32} & s_{33} & \ldots & a_{3} a_{4} \ldots a_{n-1} s_{n 3} \\
\vdots & \vdots & \vdots & \ddots & \vdots \\
s_{n 1} & s_{n 2} & s_{n 3} & \ldots & s_{n n}
\end{array}\right) .
\end{gathered}
$$

It was shown in $[15,16]$ that the set $\mathcal{A}_{a_{1}, \ldots, a_{n-1}}(n)$ with the standard commutator is a Lie algebra. This Lie algebra depends on $n-1$ parameters and is called a quasisimple orthogonal algebra. In the case, when all these parameters are nonzero, it is isomorphic to the Lie algebra $\mathfrak{s o}(n)$ or $\mathfrak{s o}(p, n-p)$. A more interesting situation is when some of the parameters are equal to zero. For instance, when $a_{1}=0$ and all others are nonzero, we get a contraction in the sense of Inönü-Wigner to the Euclidean algebra iso $(n)$ or the Poincaré algebra iso $(n-1,1)$. The infinite dimensional case was considered in papers [6,28].

In our article, we will only be interested in the finite-dimensional case, with the family of brackets given by

$$
[X, Y]_{S}=X S Y-Y S X
$$

where $X, Y \in \mathcal{A}_{a_{1}, \ldots, a_{n-1}}(n), S \in \mathcal{S}_{a_{1}, \ldots, a_{n-1}}(n)$. We denote this Lie bundle by $\left(\mathcal{A}_{a_{1}, \ldots, a_{n-1}}(n), \mathcal{S}_{a_{1}, \ldots, a_{n-1}}(n)\right)$. This case was studied in detail in [29].

The dual space $\left(\mathcal{A}_{a_{1}, \ldots, a_{n-1}}(n)\right)^{*}$ to the algebra $\mathcal{A}_{a_{1}, \ldots, a_{n-1}}(n)$ can be identified with the set of upper triangular matrices

$$
\mathcal{L}_{+}(n)=\left\{\mathbf{x}=\left(x_{i j}\right) \in M_{n}(\mathbb{R}): x_{i j}=0, \quad \text { for } \quad i \geq j\right\}
$$

with the pairing given by the trace

$$
\langle\mathbf{x}, X\rangle=\operatorname{Tr}(\mathbf{x} X) .
$$

Thus, the Lie bundle $\left(\mathcal{A}_{a_{1}, \ldots, a_{n-1}}(n), \mathcal{S}_{a_{1}, \ldots, a_{n-1}}(n)\right)$ introduces a family of Lie-Poisson brackets on the dual space $\mathcal{L}_{+}(n)$ :

$$
\{f, g\}_{S}(\mathbf{x})=\left\langle\mathbf{x},[d f(\mathbf{x}), d g(\mathbf{x})]_{S}\right\rangle .
$$

This family of brackets is indexed by $S \in \mathcal{S}_{a_{1}, \ldots, a_{n-1}}(n)$. For fixed $a_{1}, \ldots, a_{n-1}$ the Lie bracket from Equation (12) is linear in $S$, and consequently, the Lie-Poisson brackets are compatible.

There are known Casimir functions for the Lie bracket (14) in the case when $a_{1}=\ldots=a_{n-1}=1$ and $\operatorname{det} S \neq 0$ given by

$$
C_{k}(\mathbf{x})=\frac{1}{2 k} \operatorname{Tr}\left(\left(\mathbf{x}-\mathbf{x}^{T}\right) S^{-1}\right)^{2 k}, \quad k=1,2, \ldots,
$$

see [2]. In the case when parameters $a_{1}, \ldots, a_{n-1}$ are non-zero but not equal to one, this family has to be modified

$$
C_{k}(\mathbf{x})=\frac{1}{2 k} \operatorname{Tr}\left(\left(\mathbf{x}-\delta^{-1} \mathbf{x}^{T} \delta\right) S^{-1}\right)^{2 k},
$$


where $\delta=\operatorname{diag}\left(1, a_{1}, a_{1} a_{2}, \ldots, a_{1} a_{2} \ldots a_{n-1}\right)$, see [29]. In the situation when it is allowed that some of the parameters $a_{i}$ are equal to zero, we get

$$
C_{k}(\mathbf{x})=\frac{(-2)^{k}}{2 k} \operatorname{Tr}\left(\mathbf{x} S^{-1} \eta\left(S^{-1} \mathbf{x}\right)^{T} \delta\right)^{k}
$$

where $\eta=\operatorname{diag}\left(a_{1} a_{2} \ldots a_{n-1}, a_{2} \ldots a_{n-1}, \ldots, 1\right)$. However, in this case, not all of the functions have to be included.

The Lie bundle $\left(\mathcal{A}_{a_{1}, \ldots, a_{n-1}}(n), \mathcal{S}_{a_{1}, \ldots, a_{n-1}}(n)\right)$ can be generalized to the case when some parameters $a_{i}$ are equal to zero. Let us suppose that $a_{k_{1}}=\ldots=a_{k_{N}}=0$, where $k_{1}, \ldots, k_{N}$ is an increasing sequence, then both of the elements of $\mathcal{A}_{a_{1}, \ldots, a_{n-1}}(n)$ and $\mathcal{S}_{a_{1}, \ldots, a_{n-1}}(n)$ can be written in block form as

$$
X=\left(\begin{array}{c|c|c|c}
A_{0} & \mathbf{0} & \ldots & \mathbf{0} \\
\hline * & A_{1} & \ldots & \mathbf{0} \\
\hline \vdots & \vdots & \ddots & \vdots \\
\hline * & * & \ldots & A_{N}
\end{array}\right), \quad S=\left(\begin{array}{c|c|c|c}
B_{0} & \mathbf{0} & \ldots & \mathbf{0} \\
\hline * & B_{1} & \ldots & \mathbf{0} \\
\hline \vdots & \vdots & \ddots & \vdots \\
\hline * & * & \ldots & B_{N}
\end{array}\right),
$$

where $A_{i} \in \mathcal{A}_{a_{k_{i}+1}, \ldots, a_{k_{i+1}-1}}\left(k_{i+1}-k_{i}\right), B_{i} \in \mathcal{S}_{a_{k_{i}+1}, \ldots, a_{k_{i+1}-1}}\left(k_{i+1}-k_{i}\right), k_{0}=0$, and $*$ denotes arbitrary matrices of suitable sizes. If blocks $A_{i}, D_{i} \in \mathcal{A}_{a_{k_{i}+1}, \ldots, a_{k_{i+1}-1}}\left(k_{i+1}-k_{i}\right)$ and $B_{i}$ are $2 \times 2$, then it is easy to see that $\left[A_{i}, D_{i}\right]_{B_{i}}=0$. Then nothing prevents us from replacing the almost symmetric matrix (we called it quasisimple symmetric matrix) $B_{i}=\left(\begin{array}{cc}s_{11} & a_{k_{i}+1} s_{21} \\ s_{21} & s_{22}\end{array}\right)$ with any $2 \times 2$ matrix $\widetilde{B}_{i}=\left(\begin{array}{ll}s_{11} & s_{12} \\ s_{21} & s_{22}\end{array}\right)$ because the property $\left[A_{i}, D_{i}\right]_{\widetilde{B}_{i}}=0$ is still fulfilled. We will denote by $\widetilde{\mathcal{S}_{a_{1}, \ldots, a_{n}}}(n)$ the set of matrices $S$ of the form (18), where blocks $B_{i}$ in case $2 \times 2$ can be replaced by $\widetilde{B}_{i}$.

Proposition 1. A pair $\left(\mathcal{A}_{a_{1}, \ldots, a_{n}}(n), \widetilde{\mathcal{S}_{a_{1}, \ldots, a_{n}}}(n)\right)$ is a linear bundle of Lie algebras.

In this case, we will allow that matrix elements are complex numbers. It turns out that for low dimensional Lie algebras, those two families of Lie bundles, which are dependent on parameters $a_{i}$, are sufficient for the classification of Lie algebras. In the next sections, it will be shown on three- and four-dimensional Lie algebras that all of these algebras belong to the Lie bundle $\left(\mathcal{A}_{a_{1}, \ldots, a_{n}}(n), \mathcal{S}_{a_{1}, \ldots, a_{n}}(n)\right)$ or $\left(\mathcal{A}_{a_{1}, \ldots, a_{n}}(n), \widetilde{\mathcal{S}_{a_{1}, \ldots, a_{n}}}(n)\right)$.

\section{Three-Dimensional Lie Algebras and Lie Bundles}

First Lie bundle.

Let us consider the Lie algebra so(3) with the standard commutator. Note that $\mathcal{A}_{1,1}(3)$ as a set coincides with it but carries another Lie bracket

$$
\left[X_{1}, Y_{1}\right]_{S_{1}}=X_{1} S_{1} Y_{1}-X_{1} S_{1} Y_{1}
$$

where $X_{1}, Y_{1} \in \mathcal{A}_{1,1}(3)$ and $S_{1} \in \mathcal{S}_{1,1}(3)$. We assume the forms of $X_{1}$ and $S_{1}$ as follows

$$
X_{1}=\left(\begin{array}{ccc}
0 & -x_{3} & x_{2} \\
x_{3} & 0 & -x_{1} \\
-x_{2} & x_{1} & 0
\end{array}\right), \quad S_{1}=\left(\begin{array}{lll}
s_{11} & s_{21} & s_{31} \\
s_{21} & s_{22} & s_{32} \\
s_{31} & s_{32} & s_{33}
\end{array}\right) \text {. }
$$


From the definition in Equation (14), we get the Lie-Poisson bracket on the dual space $\mathcal{L}_{+}(3)$ to the Lie algebra $\mathcal{A}_{1,1}(3)$

$$
\begin{aligned}
\{f, g\}_{s_{1}}(\mathbf{x}) & =\left(s_{31} x_{1}+s_{32} x_{2}+s_{33} x_{3}\right)\left(\frac{\partial f}{\partial x_{1}}(\mathbf{x}) \frac{\partial g}{\partial x_{2}}(\mathbf{x})-\frac{\partial f}{\partial x_{2}}(\mathbf{x}) \frac{\partial g}{\partial x_{1}}(\mathbf{x})\right) \\
& +\left(s_{11} x_{1}+s_{21} x_{2}+s_{31} x_{3}\right)\left(\frac{\partial f}{\partial x_{2}}(\mathbf{x}) \frac{\partial g}{\partial x_{3}}(\mathbf{x})-\frac{\partial f}{\partial x_{3}}(\mathbf{x}) \frac{\partial g}{\partial x_{2}}(\mathbf{x})\right) \\
& +\left(s_{21} x_{1}+s_{22} x_{2}+s_{32} x_{3}\right)\left(\frac{\partial f}{\partial x_{3}}(\mathbf{x}) \frac{\partial g}{\partial x_{1}}(\mathbf{x})-\frac{\partial f}{\partial x_{1}}(\mathbf{x}) \frac{\partial g}{\partial x_{3}}(\mathbf{x})\right) .
\end{aligned}
$$

In our calculation, we took $\mathbf{x} \in \mathcal{L}_{+}(3)$ of the form

$$
\mathbf{x}=\left(\begin{array}{ccc}
0 & x_{3} & -x_{2} \\
0 & 0 & x_{1} \\
0 & 0 & 0
\end{array}\right)
$$

Thus, the matrix of the Poisson tensor assumes the following form

$$
\pi_{S_{1}}(\mathbf{x})=\left(\begin{array}{ccc}
0 & s_{31} x_{1}+s_{32} x_{2}+s_{33} x_{3} & -s_{21} x_{1}-s_{22} x_{2}-s_{32} x_{3} \\
-s_{31} x_{1}-s_{32} x_{2}-s_{33} x_{3} & 0 & s_{11} x_{1}+s_{21} x_{2}+s_{31} x_{3} \\
s_{21} x_{1}+s_{22} x_{2}+s_{32} x_{3} & -s_{11} x_{1}-s_{21} x_{2}-s_{31} x_{3} & 0
\end{array}\right) .
$$

\section{Second Lie bundle.}

We can also get another Lie-Poisson bracket

$$
\left[X_{2}, Y_{2}\right]_{S_{2}}=X_{2} S_{2} Y_{2}-Y_{2} S_{2} X_{2}
$$

by taking $X_{2}, Y_{2} \in \mathcal{A}_{1,0}(3)$ and $S_{2} \in \widetilde{\mathcal{S}_{1,0}}(3)$ as follows

$$
X_{2}=\left(\begin{array}{ccc}
0 & -x_{3} & 0 \\
x_{3} & 0 & 0 \\
-x_{2} & x_{1} & 0
\end{array}\right), \quad S_{2}=\left(\begin{array}{ccc}
s_{11} & s_{12} & 0 \\
s_{21} & s_{22} & 0 \\
s_{31} & s_{32} & s_{33}
\end{array}\right) .
$$

Using a similar calculation as in Equation (21), we get the next Lie-Poisson bracket

$$
\begin{aligned}
\{f, g\}_{S_{2}}(\mathbf{x}) & =\left(s_{11} x_{1}+s_{12} x_{2}\right)\left(\frac{\partial f}{\partial x_{2}}(\mathbf{x}) \frac{\partial g}{\partial x_{3}}(\mathbf{x})-\frac{\partial f}{\partial x_{3}}(\mathbf{x}) \frac{\partial g}{\partial x_{2}}(\mathbf{x})\right) \\
& +\left(s_{21} x_{1}+s_{11} x_{2}\right)\left(\frac{\partial f}{\partial x_{3}}(\mathbf{x}) \frac{\partial g}{\partial x_{1}}(\mathbf{x})-\frac{\partial f}{\partial x_{1}}(\mathbf{x}) \frac{\partial g}{\partial x_{3}}(\mathbf{x})\right) .
\end{aligned}
$$

Thus, the matrix of the Poisson tensor in this case is given by

$$
\pi_{S_{2}}(\mathbf{x})=\left(\begin{array}{ccc}
0 & 0 & -s_{21} x_{1}-s_{22} x_{2} \\
0 & 0 & s_{11} x_{1}+s_{12} x_{2} \\
s_{21} x_{1}+s_{22} x_{2} & -s_{11} x_{1}-s_{12} x_{2} & 0
\end{array}\right)
$$

Table 1 describes which Lie-Poisson structures that are related to the three-dimensional Lie algebras $\mathfrak{g}_{i j}$, according to the classification taken from [30], can be obtained from those two brackets. An isomorphism is given by mapping $\left(x_{1}, x_{2}, x_{3}\right) \mapsto\left(e_{1}, e_{2}, e_{3}\right)$. It is easy to see that some of them can be obtained from both Equations (19) and (23). Furthermore, these brackets split all of these three-dimensional Lie algebras into two families-algebras in the same family are all compatible with each other but not with those from the other one. 
Table 1. Three-dimensional Lie algebras with their Poisson structure (22) or (26) and corresponding matrices $S_{1}, S_{2}$ from Equations (19) and (23).

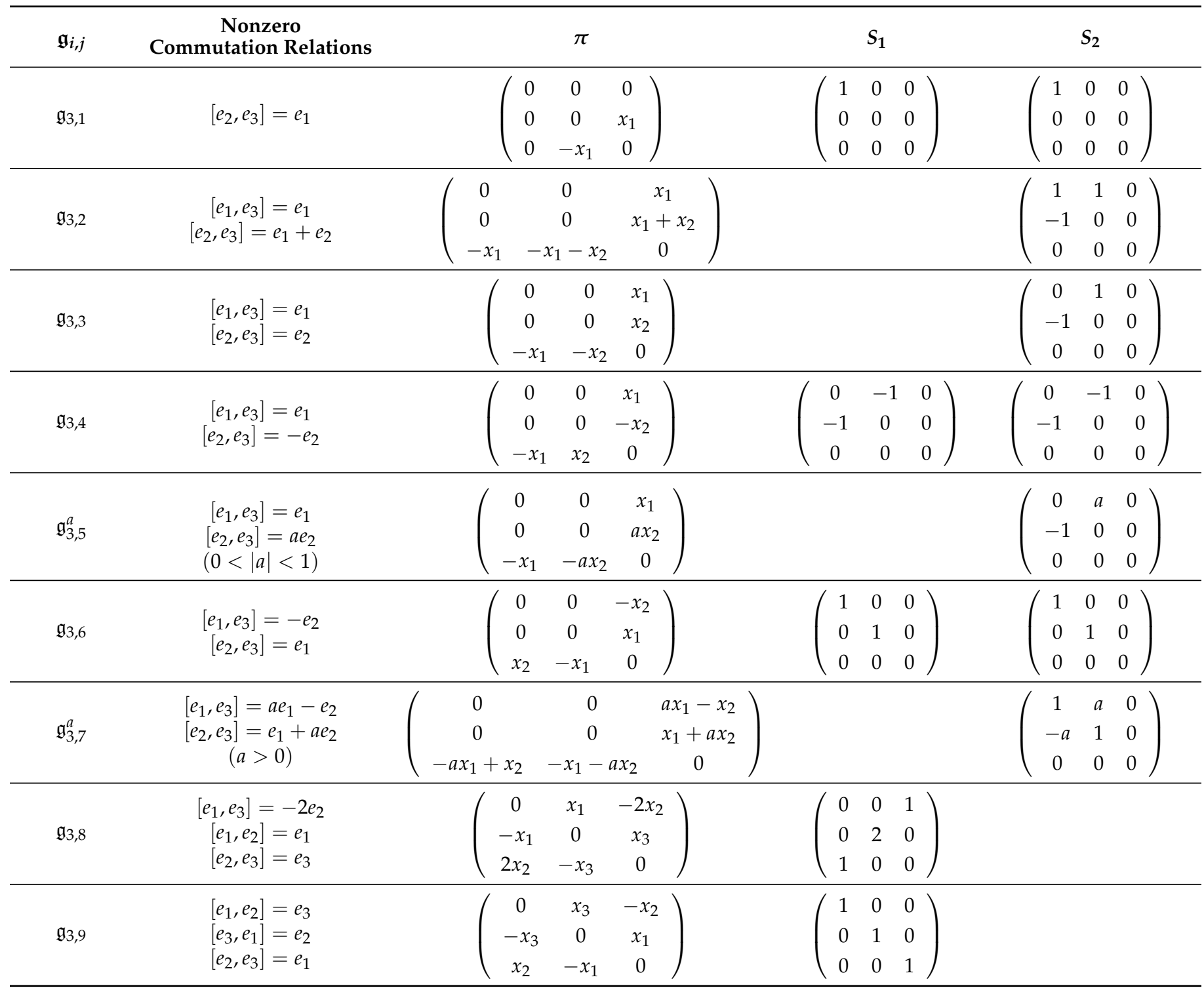

We get two families:

family I: $\quad \mathfrak{g}_{3,1}, \mathfrak{g}_{3,4}, \mathfrak{g}_{3,6}, \mathfrak{g}_{3,8}, \mathfrak{g}_{3,9}$,

family II: $\quad \mathfrak{g}_{3,1}, \mathfrak{g}_{3,2}, \mathfrak{g}_{3,3}, \mathfrak{g}_{3,4}, \mathfrak{g}_{3,5}^{a}, \mathfrak{g}_{3,6}, \mathfrak{g}_{3,7}^{a}$.

Obviously, we would get the same result by analyzing the compatibility of Lie-Poisson structures related, respectively, to those three-dimensional Lie algebras, see Table 2.

Belonging to the appropriate family of a Lie bundle also requires that Casimirs for the Poisson bracket (respectively, invariants for Lie algebras) are generally described by one function. For the first family of Lie algebras, after direct calculation from Equation (15), we get

$$
\begin{aligned}
C_{1}(\mathbf{x}) & =-\frac{\operatorname{det} S_{1}}{2} \operatorname{Tr}\left(\left(\mathbf{x}-\mathbf{x}^{T}\right) s_{1}^{-1}\right)^{2} \\
& =s_{11} x_{1}^{2}+s_{22} x_{2}^{2}+s_{33} x_{3}^{2}+2 s_{21} x_{1} x_{2}+2 s_{31} x_{1} x_{3}+2 s_{32} x_{2} x_{3} .
\end{aligned}
$$


Table 3 displays the results in some specific situations. For the second family, the Casimir functions can be obtained by solving the linear first order partial differential equation

$$
\left(s_{21} x_{1}+s_{22} x_{2}\right) \frac{\partial C_{1}}{\partial x_{1}}(\mathbf{x})-\left(s_{11} x_{1}+s_{12} x_{2}\right) \frac{\partial C_{1}}{\partial x_{2}}(\mathbf{x})=0
$$

Table 2. Three-dimensional Lie algebras and bi-Hamiltonian structures on $\mathcal{L}_{+}(3)$. Symbol + means that the Lie algebras from the corresponding row and column are compatible and symbol - that they are not compatible.

\begin{tabular}{|c|c|c|c|c|c|c|c|c|c|}
\hline $\mathfrak{g}_{3,1}$ & $\mathfrak{g}_{3,2}$ & $\mathfrak{g}_{3,3}$ & $\mathfrak{g}_{3,4}$ & $\mathfrak{g}_{3,5}^{a}$ & $\mathfrak{g}_{3,6}$ & $\mathfrak{g}_{3,7}^{a}$ & $\mathfrak{g}_{3,8}$ & $\mathfrak{g}_{3,9}$ & Name \\
\hline \multirow[t]{9}{*}{+} & + & + & + & + & + & + & + & + & $\mathfrak{g}_{3,1}$ \\
\hline & + & + & + & + & + & + & - & - & $\mathfrak{g}_{3,2}$ \\
\hline & & + & + & + & + & + & - & - & $\mathfrak{g}_{3,3}$ \\
\hline & & & + & + & + & + & + & + & $\mathfrak{g}_{3,4}$ \\
\hline & & & & + & + & + & - & - & $\mathfrak{g}_{3,5}^{a}$ \\
\hline & & & & & + & + & + & + & $\mathfrak{g}_{3,6}$ \\
\hline & & & & & & + & - & - & $\mathfrak{g}_{3,7}^{a}$ \\
\hline & & & & & & & + & + & $\mathfrak{g}_{3,8}$ \\
\hline & & & & & & & & + & $\mathfrak{g}_{3,9}$ \\
\hline
\end{tabular}

Table 3. Casimir functions for the first family of three-dimensional Lie algebras.

\begin{tabular}{ccc}
\hline $\mathfrak{g}_{i, j}$ & $\left(\begin{array}{lll}1 & 0 & 0 \\
0 & 0 & 0 \\
0 & 0 & 0\end{array}\right)$ & Invariants \\
\hline $\mathfrak{g}_{3,1}$ & $\left(\begin{array}{ccc}0 & -1 & 0 \\
-1 & 0 & 0 \\
0 & 0 & 0\end{array}\right)$ & $-x_{1}^{2} x_{2}$ \\
\hline $\mathfrak{g}_{3,4}$ & $\left(\begin{array}{lll}1 & 0 & 0 \\
0 & 1 & 0 \\
0 & 0 & 0\end{array}\right)$ & \\
\hline $\mathfrak{g}_{3,6}$ & $\left(\begin{array}{lll}0 & 0 & 1 \\
0 & 2 & 0 \\
1 & 0 & 0\end{array}\right)$ & $x_{1}^{2}+x_{2}^{2}$ \\
\hline $\mathfrak{g}_{3,8}$ & $\left(\begin{array}{lll}1 & 0 & 0 \\
0 & 1 & 0 \\
0 & 0 & 1\end{array}\right)$ & $2 x_{2}^{2}+2 x_{1} x_{3}$ \\
\hline $\mathfrak{g}_{3,9}$ &
\end{tabular}

\section{Four-Dimensional Lie Algebras and Lie Bundles}

Let us consider all real Lie algebras of dimension equal to four. A complete list of these algebras is given, for example, by Mubarakzyanov [22]. There are twelve real algebras of dimension four, and five of them depend on parameters. Our list of algebras gathered in Table 4 is based on the article [30].

On the dual space $\mathcal{L}_{+}(4)$ of Lie algebras $\mathfrak{g}_{4, i}, i=1, \ldots, 12$, there are Poisson structures defined by Poisson tensors $\pi_{4, i}$.

Table 5 describes if the above structures are compatible in sense (8), i.e., $\mathbb{R}^{4}$ equipped with these is a bi-Hamiltonian manifold. From this table, we can see that we have three families of compatible structures, which are: 
family I: $\quad \mathfrak{g}_{4,1}, \mathfrak{g}_{4,3}, \mathfrak{g}_{4,12}$,

family II: $\quad \mathfrak{g}_{4,1}, \mathfrak{g}_{4,2}^{a}, \mathfrak{g}_{4,3}, \mathfrak{g}_{4,4}, \mathfrak{g}_{4,5}^{a b}, \mathfrak{g}_{4,6}^{a b}$

family III: $\quad \mathfrak{g}_{4,1}, \mathfrak{g}_{4,7}, \mathfrak{g}_{4,8}, \mathfrak{g}_{4,9}^{b}, \mathfrak{g}_{4,10}, \mathfrak{g}_{4,11}^{a}, \mathfrak{g}_{4,2}^{2}, \mathfrak{g}_{4,5}^{1-b b}, \mathfrak{g}_{4,6}^{2 b b}$.

Table 4. Real Lie algebras of dimension four.

\begin{tabular}{|c|c|}
\hline Name & Nonzero Commutation Relations \\
\hline $\mathfrak{g}_{4,1}$ & {$\left[e_{2}, e_{4}\right]=e_{1}, \quad\left[e_{3}, e_{4}\right]=e_{2}$} \\
\hline $\mathfrak{g}_{4,2}^{a}$ & {$\left[e_{1}, e_{4}\right]=a e_{1}, \quad\left[e_{2}, e_{4}\right]=e_{2}, \quad\left[e_{3}, e_{4}\right]=e_{2}+e_{3}, \quad(a \neq 0)$} \\
\hline $\mathfrak{g}_{4,3}$ & {$\left[e_{1}, e_{4}\right]=e_{1}, \quad\left[e_{3}, e_{4}\right]=e_{2}$} \\
\hline $\mathfrak{g}_{4,4}$ & {$\left[e_{1}, e_{4}\right]=e_{1}, \quad\left[e_{2}, e_{4}\right]=e_{1}+e_{2}, \quad\left[e_{3}, e_{4}\right]=e_{2}+e_{3}$} \\
\hline $\mathfrak{g}_{4,5}^{a b}$ & $\begin{array}{c}{\left[e_{1}, e_{4}\right]=e_{1}, \quad\left[e_{2}, e_{4}\right]=a e_{2}} \\
{\left[e_{3}, e_{4}\right]=b e_{3} \quad(a b \neq 0, \quad-1 \leq a \leq b \leq 1)}\end{array}$ \\
\hline $\mathfrak{g}_{4,6}^{a b}$ & $\begin{array}{l}{\left[e_{1}, e_{4}\right]=a e_{1}, \quad\left[e_{2}, e_{4}\right]=b e_{2}-e_{3}} \\
{\left[e_{3}, e_{4}\right]=e_{2}+b e_{3}, \quad(a \neq 0, \quad b \geq 0)}\end{array}$ \\
\hline $\mathfrak{g}_{4,7}$ & {$\left[e_{2}, e_{3}\right]=e_{1}, \quad\left[e_{1}, e_{4}\right]=2 e_{1}, \quad\left[e_{2}, e_{4}\right]=e_{2}, \quad\left[e_{3}, e_{4}\right]=e_{2}+e_{3}$} \\
\hline $\mathfrak{g}_{4,8}$ & {$\left[e_{2}, e_{3}\right]=e_{1}, \quad\left[e_{2}, e_{4}\right]=e_{2}, \quad\left[e_{3}, e_{4}\right]=-e_{3}$} \\
\hline $\mathfrak{g}_{4,9}^{b}$ & $\begin{array}{c}{\left[e_{2}, e_{3}\right]=e_{1}, \quad\left[e_{1}, e_{4}\right]=(1+b) e_{1}} \\
{\left[e_{2}, e_{4}\right]=e_{2}, \quad\left[e_{3}, e_{4}\right]=b e_{3}, \quad(-1<b \leq 1)}\end{array}$ \\
\hline $\mathfrak{g}_{4,10}$ & {$\left[e_{2}, e_{3}\right]=e_{1}, \quad\left[e_{2}, e_{4}\right]=-e_{3}, \quad\left[e_{3}, e_{4}\right]=e_{2}$} \\
\hline $\mathfrak{g}_{4,11}^{a}$ & $\begin{aligned} & {\left[e_{2}, e_{3}\right]=e_{1}, \quad\left[e_{1}, e_{4}\right]=2 a e_{1}, } \\
{\left[e_{2}, e_{4}\right]=} & a e_{2}-e_{3}, \quad\left[e_{3}, e_{4}\right]=e_{2}+a e_{3}, \quad(a>0)\end{aligned}$ \\
\hline $\mathfrak{g}_{4,12}$ & {$\left[e_{1}, e_{3}\right]=e_{1}, \quad\left[e_{2}, e_{3}\right]=e_{2}, \quad\left[e_{1}, e_{4}\right]=-e_{2}, \quad\left[e_{2}, e_{4}\right]=e_{1}$} \\
\hline
\end{tabular}

Table 5. Four-dimensional Lie algebras and bi-Hamiltonian structures on $\mathcal{L}_{+}(4)$. Symbol + means that the Lie algebras from the corresponding row and column are compatible, symbol - that they are not compatible; the symbol $-{ }^{*}$ means that they are not compatible unless $a=2$ for Lie algebra $\mathfrak{g}_{4,2}^{a}$. Moreover, symbols $-^{* *}$ and $-^{* * *}$ mean that the corresponding Lie algebras are compatible only if $a+b=1$ for Lie algebra $\mathfrak{g}_{4,5}^{a b}$ or $a=2 b$ for Lie algebra $\mathfrak{g}_{4,6}^{a b}$, respectively.

\begin{tabular}{|c|c|c|c|c|c|c|c|c|c|c|c|c|}
\hline $\mathfrak{g}_{4,1}$ & $\mathfrak{g}_{4,2}^{a}$ & $\mathfrak{g}_{4,3}$ & $\mathfrak{g}_{4,4}$ & $\mathfrak{g}_{4,5}^{a b}$ & $\mathfrak{g}_{4,6}^{a b}$ & $\mathfrak{g}_{4,7}$ & $\mathfrak{g}_{4,8}$ & $\mathfrak{g}_{4,9}^{b}$ & $\mathfrak{g}_{4,10}$ & $\mathfrak{g}_{4,11}^{a}$ & $\mathfrak{g}_{4,12}$ & Name \\
\hline \multirow[t]{12}{*}{+} & + & + & + & + & + & + & + & + & + & + & + & $\mathfrak{g}_{4,1}$ \\
\hline & + & + & + & + & + & $-^{*}$ & $-^{*}$ & $-^{*}$ & $-^{*}$ & $-^{*}$ & - & $\mathfrak{g}_{4,2}^{a}$ \\
\hline & & + & + & + & + & - & - & - & - & - & + & $\mathfrak{g}_{4,3}$ \\
\hline & & & + & + & + & - & - & - & - & - & - & $\mathfrak{g}_{4,4}$ \\
\hline & & & & + & + & $-^{* *}$ & $-^{* *}$ & $-^{* *}$ & $-^{* *}$ & $-^{* *}$ & - & $\mathfrak{g}_{4,5}^{a b}$ \\
\hline & & & & & + & $-{ }^{* * *}$ & $-{ }^{* * *}$ & $-{ }^{* * *}$ & $-{ }^{* * *}$ & $-{ }^{* * *}$ & - & $\mathfrak{g}_{4,6}^{a b}$ \\
\hline & & & & & & + & + & + & + & + & - & $\mathfrak{g}_{4,7}$ \\
\hline & & & & & & & + & + & + & + & - & $\mathfrak{g}_{4,8}$ \\
\hline & & & & & & & & + & + & + & - & $\mathfrak{g}_{4,9}^{b}$ \\
\hline & & & & & & & & & + & + & - & $\mathfrak{g}_{4,10}$ \\
\hline & & & & & & & & & & + & - & $\mathfrak{g}_{4,11}^{a}$ \\
\hline & & & & & & & & & & & + & $\mathfrak{g}_{4,12}$ \\
\hline
\end{tabular}

First Lie bundle.

The first family can be obtain by taking the bracket

$$
\left[X_{1}, Y_{1}\right]_{S_{1}}=X_{1} S_{1} Y_{1}-Y_{1} S_{1} X_{1}
$$

where $X_{1}, Y_{1} \in \mathcal{A}_{1,0,1}(4)$ and $S_{1} \in \widetilde{\mathcal{S}_{1,0,1}}(4)$ are matrices of the form

$$
X_{1}=\left(\begin{array}{cccc}
0 & -x_{3} & 0 & 0 \\
x_{3} & 0 & 0 & 0 \\
-x_{2} & x_{1} & 0 & y_{3} \\
-y_{1} & -y_{2} & -y_{3} & 0
\end{array}\right), \quad s_{1}=\left(\begin{array}{cccc}
s_{11} & s_{12} & 0 & 0 \\
s_{21} & s_{22} & 0 & 0 \\
s_{31} & s_{32} & s_{33} & s_{34} \\
s_{41} & s_{42} & s_{43} & s_{44}
\end{array}\right) .
$$


After direct calculation from Equation (14), we get the following formula

$$
\begin{aligned}
\{f, g\}_{S_{1}}(\mathbf{x}) & =\left(\frac{\partial f}{\partial x_{3}}(\mathbf{x}) \frac{\partial g}{\partial x_{1}}(\mathbf{x})-\frac{\partial f}{\partial x_{1}}(\mathbf{x}) \frac{\partial g}{\partial x_{3}}(\mathbf{x})\right)\left(s_{22} x_{2}+s_{21} x_{1}\right) \\
& +\left(\frac{\partial f}{\partial x_{3}}(\mathbf{x}) \frac{\partial g}{\partial y_{2}}(\mathbf{x})-\frac{\partial f}{\partial y_{2}}(\mathbf{x}) \frac{\partial g}{\partial x_{3}}(\mathbf{x})\right)\left(s_{21} y_{2}-s_{22} y_{1}\right) \\
& +\left(\frac{\partial f}{\partial y_{2}}(\mathbf{x}) \frac{\partial g}{\partial y_{3}}(\mathbf{x})-\frac{\partial f}{\partial y_{3}}(\mathbf{x}) \frac{\partial g}{\partial y_{2}}(\mathbf{x})\right)\left(s_{34} y_{2}+s_{44} x_{1}\right) \\
& +\left(\frac{\partial f}{\partial y_{3}}(\mathbf{x}) \frac{\partial g}{\partial x_{1}}(\mathbf{x})-\frac{\partial f}{\partial x_{1}}(\mathbf{x}) \frac{\partial g}{\partial y_{3}}(\mathbf{x})\right)\left(s_{33} y_{2}+s_{43} x_{1}\right) \\
& +\left(\frac{\partial f}{\partial x_{3}}(\mathbf{x}) \frac{\partial g}{\partial y_{1}}(\mathbf{x})-\frac{\partial f}{\partial y_{1}}(\mathbf{x}) \frac{\partial g}{\partial x_{3}}(\mathbf{x})\right)\left(s_{11} y_{2}-s_{12} y_{1}\right) \\
& +\left(\frac{\partial f}{\partial y_{1}}(\mathbf{x}) \frac{\partial g}{\partial y_{3}}(\mathbf{x})-\frac{\partial f}{\partial y_{3}}(\mathbf{x}) \frac{\partial g}{\partial y_{1}}(\mathbf{x})\right)\left(s_{34} y_{1}-s_{44} x_{2}\right) \\
& +\left(\frac{\partial f}{\partial x_{2}}(\mathbf{x}) \frac{\partial g}{\partial y_{3}}(\mathbf{x})-\frac{\partial f}{\partial y_{3}}(\mathbf{x}) \frac{\partial g}{\partial x_{2}}(\mathbf{x})\right)\left(s_{33} y_{1}-s_{43} x_{2}\right) \\
& +\left(\frac{\partial f}{\partial x_{2}}(\mathbf{x}) \frac{\partial g}{\partial x_{3}}(\mathbf{x})-\frac{\partial f}{\partial x_{3}}(\mathbf{x}) \frac{\partial g}{\partial x_{2}}(\mathbf{x})\right)\left(s_{12} x_{2}+s_{11} x_{1}\right) \\
& +\left(\frac{\partial f}{\partial x_{3}}(\mathbf{x}) \frac{\partial g}{\partial y_{3}}(\mathbf{x})-\frac{\partial f}{\partial y_{3}}(\mathbf{x}) \frac{\partial g}{\partial x_{3}}(\mathbf{x})\right)\left(s_{42} x_{2}-s_{32} y_{1}+s_{31} y_{2}+s_{41} x_{1}\right)
\end{aligned}
$$

where $\mathbf{x} \in \mathcal{L}_{+}(4)$ is an upper triangular matrix given by

$$
\mathbf{x}=\left(\begin{array}{cccc}
0 & x_{3} & -x_{2} & -y_{1} \\
0 & 0 & x_{1} & -y_{2} \\
0 & 0 & 0 & -y_{3} \\
0 & 0 & 0 & 0
\end{array}\right)
$$

As we can see, we obtained six-dimensional cases. To get our four-dimensional families, we limit ourselves to variables $\left(x_{2}, x_{3}, y_{1}, y_{3}\right)$ by putting $x_{1}=y_{2}=0$ and $s_{11}=$ $s_{31}=s_{41}=0$. This means restricting to the subspaces $V \subset \mathcal{A}_{1,0,1}(4)$ and $\mathcal{S} \subset \widetilde{\mathcal{S}_{1,0,1}}(4)$, whose elements are given by

$$
\widetilde{X_{1}}=\left(\begin{array}{cccc}
0 & -x_{3} & 0 & 0 \\
x_{3} & 0 & 0 & 0 \\
-x_{2} & 0 & 0 & y_{3} \\
-y_{1} & 0 & -y_{3} & 0
\end{array}\right), \quad \widetilde{s_{1}}=\left(\begin{array}{cccc}
0 & s_{12} & 0 & 0 \\
s_{21} & s_{22} & 0 & 0 \\
0 & s_{32} & s_{33} & s_{34} \\
0 & s_{42} & s_{43} & s_{44}
\end{array}\right) .
$$

Then we get a four-dimensional Lie subalgebra with bracket

$$
\left[\widetilde{X_{1}}, \widetilde{Y}_{1}\right]_{\widetilde{S_{1}}}=\widetilde{X_{1}} \widetilde{S_{1}} \widetilde{Y}_{1}-\widetilde{Y_{1}} \widetilde{S_{1}} \widetilde{X_{1}}
$$

We get the Lie-Poisson structure related to the Lie algebra $\mathfrak{g}_{4,1}$ by putting $s_{44}=s_{32}=-1$ and the other parameters equal to zero in matrix $\widetilde{s}_{1}$. To get the Lie-Poisson structure associated with the Lie algebra $\mathfrak{g}_{4,3}$ in matrix $\widetilde{s_{1}}$, we put $s_{32}=s_{43}=-1$ and all others parameters equal to zero. To get the Lie-Poisson structure associated with Lie algebra $\mathfrak{g}_{4,12}$, we put $s_{33}=s_{44}=-1, s_{12}=1$ and other parameters equal to zero in matrix $\widetilde{S_{1}}$. In these cases, an isomorphism is given by the mapping $\left(x_{2}, y_{1}, x_{3}, y_{3}\right) \mapsto\left(e_{1}, e_{2}, e_{3}, e_{4}\right)$.

In Table 6, there are Lie-Poisson structures related to four-dimensional Lie algebras $\mathfrak{g}_{4, i}$ from the first family that can be obtained from bracket (31) by choosing a suitable matrix $\widetilde{S_{1}}$ and putting $x_{1}=y_{2}=0$. 
Table 6. First Lie bundle for four-dimensional Lie algebras.

\begin{tabular}{|c|c|c|c|c|c|c|c|c|}
\hline $\mathfrak{g}_{i, j}$ & \multicolumn{4}{|c|}{$\pi$} & \multicolumn{4}{|c|}{$\widetilde{S_{1}}$} \\
\hline \multirow{4}{*}{$\mathfrak{g}_{4,1}$} & 10 & 0 & 0 & 0 & $(0$ & 0 & 0 & 0 \\
\hline & 0 & 0 & 0 & $x_{2}$ & 0 & 0 & 0 & 0 \\
\hline & 0 & 0 & 0 & $y_{1}$ & 0 & -1 & 0 & 0 \\
\hline & 0 & $-x_{2}$ & $-y_{1}$ & 0 & 0 & 0 & 0 & -1 \\
\hline \multirow{4}{*}{$\mathfrak{g}_{4,3}$} & $(0$ & 0 & 0 & $x_{2}$ & $(0$ & 0 & 0 & 0 \\
\hline & 0 & 0 & 0 & 0 & 0 & 0 & 0 & 0 \\
\hline & 0 & 0 & 0 & $y_{1}$ & 0 & -1 & 0 & 0 \\
\hline & $-x_{2}$ & 20 & $-y_{1}$ & 0 & 0 & 0 & -1 & 0 \\
\hline \multirow{4}{*}{$\mathfrak{g}_{4,12}$} & 0 & 0 & $x_{2}$ & $-y_{1}$ & $(0$ & 1 & 0 & 0 \\
\hline & 0 & 0 & $y_{1}$ & $x_{2}$ & 0 & 0 & 0 & 0 \\
\hline & $-x_{2}$ & $-y_{1}$ & 0 & 0 & 0 & 0 & -1 & 0 \\
\hline & $y_{1}$ & $-x_{2}$ & 0 & 0 & 0 & 0 & 0 & -1 \\
\hline
\end{tabular}

\section{Second Lie bundle.}

The second family can be obtained by taking the bracket

$$
\left[X_{2}, Y_{2}\right]_{S_{2}}=X_{2} S_{2} Y_{2}-Y_{2} S_{2} X_{2}
$$

where $X_{2}, Y_{2} \in \mathcal{A}_{0,0,1}(4), S_{2} \in \widetilde{\mathcal{S}_{1,0,1}}(4)$ are matrices of the form

$$
X_{2}=\left(\begin{array}{cccc}
0 & 0 & 0 & 0 \\
x_{3} & 0 & 0 & 0 \\
-x_{2} & x_{1} & 0 & y_{3} \\
-y_{1} & -y_{2} & -y_{3} & 0
\end{array}\right), \quad S_{2}=\left(\begin{array}{cccc}
s_{11} & s_{12} & 0 & 0 \\
s_{21} & s_{22} & 0 & 0 \\
s_{31} & s_{32} & s_{33} & s_{34} \\
s_{41} & s_{42} & s_{43} & s_{44}
\end{array}\right) .
$$

After direct calculation similar to Equation (31), we obtain the following formula

$$
\begin{aligned}
\{f, g\}_{S_{2}}(\mathbf{x}) & =\left(\frac{\partial f}{\partial x_{3}}(\mathbf{x}) \frac{\partial g}{\partial y_{3}}(\mathbf{x})-\frac{\partial f}{\partial y_{3}}(\mathbf{x}) \frac{\partial g}{\partial x_{3}}(\mathbf{x})\right)\left(s_{42} x_{2}-s_{32} y_{1}\right) \\
& +\left(\frac{\partial f}{\partial y_{2}}(\mathbf{x}) \frac{\partial g}{\partial x_{3}}(\mathbf{x})-\frac{\partial f}{\partial x_{3}}(\mathbf{x}) \frac{\partial g}{\partial y_{2}}(\mathbf{x})\right) s_{22} y_{1} \\
& +\left(\frac{\partial f}{\partial y_{2}}(\mathbf{x}) \frac{\partial g}{\partial y_{3}}(\mathbf{x})-\frac{\partial f}{\partial y_{3}}(\mathbf{x}) \frac{\partial g}{\partial y_{2}}(\mathbf{x})\right)\left(s_{34} y_{2}+s_{44} x_{1}\right) \\
& +\left(\frac{\partial f}{\partial y_{3}}(\mathbf{x}) \frac{\partial g}{\partial x_{1}}(\mathbf{x})-\frac{\partial f}{\partial x_{1}}(\mathbf{x}) \frac{\partial g}{\partial y_{3}}(\mathbf{x})\right)\left(s_{33} y_{2}+s_{43} x_{1}\right) \\
& +\left(\frac{\partial f}{\partial y_{1}}(\mathbf{x}) \frac{\partial g}{\partial x_{3}}(\mathbf{x})-\frac{\partial f}{\partial x_{3}}(\mathbf{x}) \frac{\partial g}{\partial y_{1}}(\mathbf{x})\right) s_{12} y_{1} \\
& +\left(\frac{\partial f}{\partial y_{1}}(\mathbf{x}) \frac{\partial g}{\partial y_{3}}(\mathbf{x})-\frac{\partial f}{\partial y_{3}}(\mathbf{x}) \frac{\partial g}{\partial y_{1}}(\mathbf{x})\right)\left(s_{34} y_{1}-s_{44} x_{2}\right) \\
& +\left(\frac{\partial f}{\partial x_{2}}(\mathbf{x}) \frac{\partial g}{\partial y_{3}}(\mathbf{x})-\frac{\partial f}{\partial y_{3}}(\mathbf{x}) \frac{\partial g}{\partial x_{2}}(\mathbf{x})\right)\left(s_{33} y_{1}-s_{43} x_{2}\right) \\
& +\left(\frac{\partial f}{\partial x_{2}}(\mathbf{x}) \frac{\partial g}{\partial x_{3}}(\mathbf{x})-\frac{\partial f}{\partial x_{3}}(\mathbf{x}) \frac{\partial g}{\partial x_{2}}(\mathbf{x})\right) s_{12} x_{2} \\
& +\left(\frac{\partial f}{\partial x_{3}}(\mathbf{x}) \frac{\partial g}{\partial x_{1}}(\mathbf{x})-\frac{\partial f}{\partial x_{1}}(\mathbf{x}) \frac{\partial g}{\partial x_{3}}(\mathbf{x})\right) s_{22} x_{2},
\end{aligned}
$$

where $\mathbf{x} \in \mathcal{L}_{+}(4)$ is defined in Equation (32). As we can see, we obtained a six-dimensional case.

We recognize in (37) the Lie-Poisson structure related to $\mathfrak{g}_{4,1}$ if we put $s_{32}=s_{44}=$ -1 and other parameters equal to zero in matrix $S_{2}$ given by Equation (36). Similarly, putting in matrix $S_{2}$ as nonzero elements only $s_{32}=s_{43}=-1$ and $x_{1}=y_{2}=0$, we 
get the Lie-Poisson structure associated with $\mathfrak{g}_{4,3}$. In both these cases, an isomorphism is given by the mapping $\left(x_{2}, y_{1}, x_{3}, y_{3}\right) \mapsto\left(e_{1}, e_{2}, e_{3}, e_{4}\right)$. Moreover, in Equation (37), we can find the Lie-Poisson structures related to $\mathfrak{g}_{4,2}^{a}$ and $\mathfrak{g}_{4,5}^{a, b}$ by a suitable choice of the matrix $S_{2}$ and putting $x_{3}=y_{3}, y_{2}=0$, see Table 7 . In these cases, an isomorphism is given by $\left(x_{1}, x_{2}, y_{1}, y_{3}\right) \mapsto\left(e_{1}, e_{2}, e_{3}, e_{4}\right)$. If we take the isomorphism $\left(e_{1}, e_{2}, e_{3}, e_{4}\right) \mapsto$ $\left(2 x_{2}+2 y_{1},-x_{1}-y_{1}+y_{2},-y_{2}, x_{3}+y_{3}\right)$ and as nonzero elements in matrix $S_{2}$, we put $s_{22}=s_{33}=s_{44}=1, s_{43}=-2$ and $x_{3}=y_{3}, x_{1}=-\tilde{x}_{1}, x_{2}=2 \tilde{x}_{2}, y_{1}=2 \tilde{x}_{2}-\tilde{x}_{1}, y_{2}=\tilde{x}_{1}-\tilde{y}_{2}$, then bracket (35) gives us the algebra $\mathfrak{g}_{4,4}$. We get the Lie algebra $\mathfrak{g}_{4,6}^{a b}$ taking matrix $S_{2}$, as in Table 7, and putting $x_{3}=y_{3}, x_{2}=x_{1}+\tilde{x}_{2}+\tilde{y}_{1}, y_{1}=x_{1}+i\left(\tilde{y}_{1}-\tilde{x}_{2}\right)$. In this case, an isomorphism is given by $\left(e_{1}, e_{2}, e_{3}, e_{4}\right) \mapsto\left(x_{1}+x_{2}+y_{1}-y_{2}, x_{2}-i y_{1}, x_{2}+i y_{1}, x_{3}+y_{3}\right)$. Similar to previous families, we get also matrix representation of these algebras, see the fourth column of Table 7. We get an analogue of Ado's theorem, see [31,32].

Table 7. Second Lie bundle for four-dimensional Lie algebras. We put $A=a\left(x_{1}+x_{2}+y_{1}-y_{2}\right), B=b\left(x_{2}-i y_{1}\right)-x_{2}-i y_{1}$, $C=x_{2}-i y_{1}+b\left(x_{2}+i y_{1}\right)$ and $D=-x_{1}+2 x_{2}+y_{1}+y_{2}$.

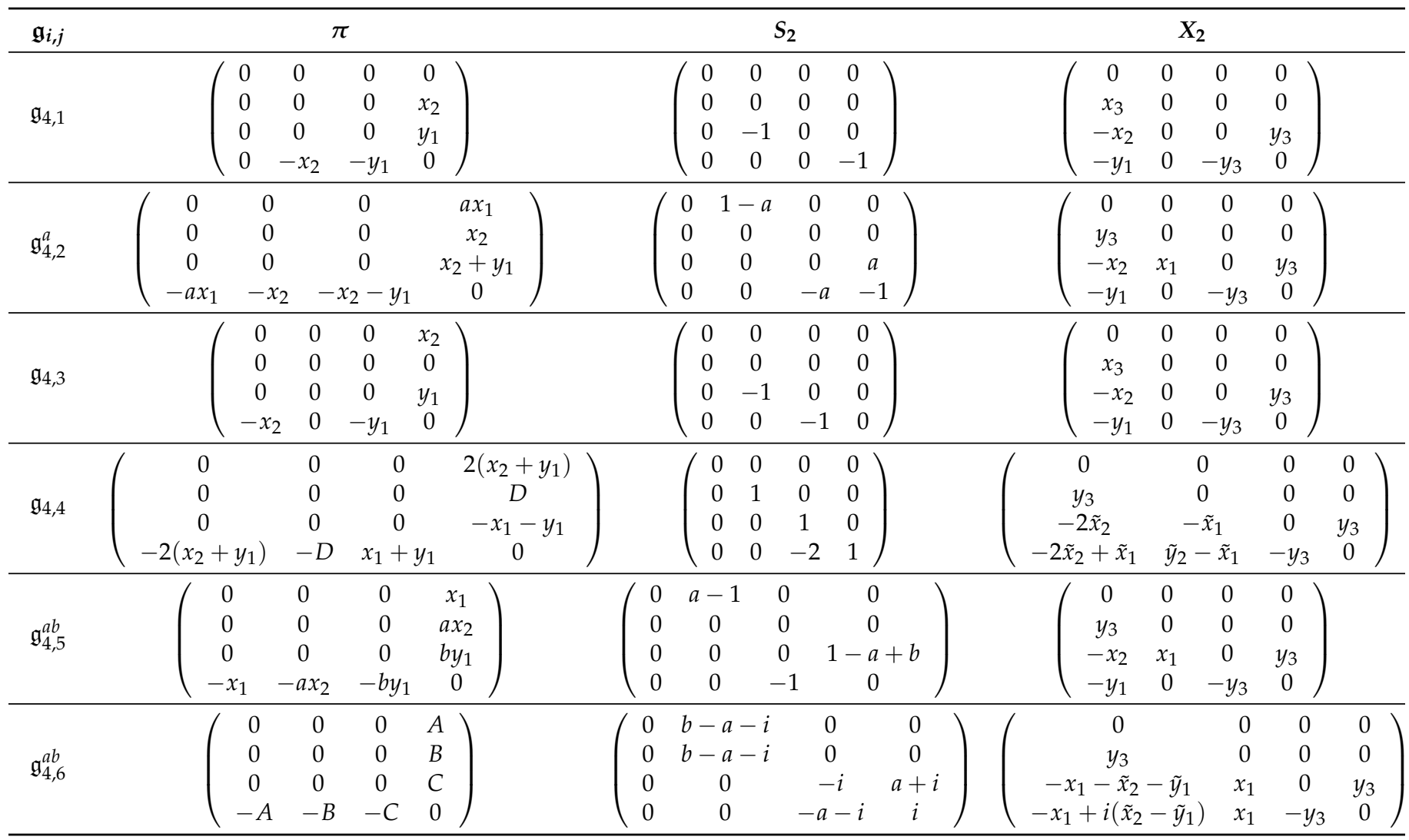

Third Lie bundle.

The third family is more complicated. The first part can be obtained by taking bracket

$$
\left[X_{3}, Y_{3}\right]_{S_{3}}=X_{3} S_{3} Y_{3}-Y_{3} S_{3} X_{3}
$$

where $X_{3}, Y_{3} \in \mathcal{A}_{0,1,1}(4)$ and $S_{3} \in \mathcal{S}_{0,1,1}(4)$ assume following forms

$$
X_{3}=\left(\begin{array}{cccc}
0 & 0 & 0 & 0 \\
x_{3} & 0 & -x_{1} & y_{2} \\
-x_{2} & x_{1} & 0 & y_{3} \\
-y_{1} & -y_{2} & -y_{3} & 0
\end{array}\right), \quad s_{3}=\left(\begin{array}{cccc}
s_{11} & 0 & 0 & 0 \\
s_{21} & s_{22} & s_{32} & s_{42} \\
s_{31} & s_{32} & s_{33} & s_{43} \\
s_{41} & s_{42} & s_{43} & s_{44}
\end{array}\right) \text {. }
$$


After direct calculation from Equation (14), we get the following Lie-Poisson bracket

$$
\begin{aligned}
\{f, g\}_{S_{3}}(\mathbf{x}) & =\left(\frac{\partial f}{\partial y_{3}}(\mathbf{x}) \frac{\partial g}{\partial x_{2}}(\mathbf{x})-\frac{\partial f}{\partial x_{2}}(\mathbf{x}) \frac{\partial g}{\partial y_{3}}(\mathbf{x})\right)\left(s_{43} x_{2}-s_{33} y_{1}\right) \\
& +\left(\frac{\partial f}{\partial x_{2}}(\mathbf{x}) \frac{\partial g}{\partial y_{2}}(\mathbf{x})-\frac{\partial f}{\partial y_{2}}(\mathbf{x}) \frac{\partial g}{\partial x_{2}}(\mathbf{x})\right)\left(s_{32} y_{1}+s_{43} x_{3}\right) \\
& +\left(\frac{\partial f}{\partial y_{3}}(\mathbf{x}) \frac{\partial g}{\partial y_{1}}(\mathbf{x})-\frac{\partial f}{\partial y_{1}}(\mathbf{x}) \frac{\partial g}{\partial y_{3}}(\mathbf{x})\right)\left(s_{44} x_{2}-s_{43} y_{1}\right) \\
& +\left(\frac{\partial f}{\partial x_{1}}(\mathbf{x}) \frac{\partial g}{\partial y_{1}}(\mathbf{x})-\frac{\partial f}{\partial y_{1}}(\mathbf{x}) \frac{\partial g}{\partial x_{1}}(\mathbf{x})\right)\left(s_{43} x_{3}+s_{42} x_{2}\right) \\
& +\left(\frac{\partial f}{\partial x_{3}}(\mathbf{x}) \frac{\partial g}{\partial y_{3}}(\mathbf{x})-\frac{\partial f}{\partial y_{3}}(\mathbf{x}) \frac{\partial g}{\partial x_{3}}(\mathbf{x})\right)\left(s_{42} x_{2}-s_{32} y_{1}\right) \\
& +\left(\frac{\partial f}{\partial y_{2}}(\mathbf{x}) \frac{\partial g}{\partial x_{3}}(\mathbf{x})-\frac{\partial f}{\partial x_{3}}(\mathbf{x}) \frac{\partial g}{\partial y_{2}}(\mathbf{x})\right)\left(s_{22} y_{1}+s_{42} x_{3}\right) \\
& +\left(\frac{\partial f}{\partial y_{1}}(\mathbf{x}) \frac{\partial g}{\partial y_{2}}(\mathbf{x})-\frac{\partial f}{\partial y_{2}}(\mathbf{x}) \frac{\partial g}{\partial y_{1}}(\mathbf{x})\right)\left(s_{42} y_{1}+s_{44} x_{3}\right) \\
& +\left(\frac{\partial f}{\partial x_{1}}(\mathbf{x}) \frac{\partial g}{\partial x_{2}}(\mathbf{x})-\frac{\partial f}{\partial x_{2}}(\mathbf{x}) \frac{\partial g}{\partial x_{1}}(\mathbf{x})\right)\left(s_{32} x_{2}+s_{33} x_{3}\right) \\
& +\left(\frac{\partial f}{\partial x_{3}}(\mathbf{x}) \frac{\partial g}{\partial x_{1}}(\mathbf{x})-\frac{\partial f}{\partial x_{1}}(\mathbf{x}) \frac{\partial g}{\partial x_{3}}(\mathbf{x})\right)\left(s_{22} x_{2}+s_{32} x_{3}\right) \\
& +\left(\frac{\partial f}{\partial x_{1}}(\mathbf{x}) \frac{\partial g}{\partial y_{3}}(\mathbf{x})-\frac{\partial f}{\partial y_{3}}(\mathbf{x}) \frac{\partial g}{\partial x_{1}}(\mathbf{x})\right)\left(s_{32} y_{3}-s_{33} y_{2}-s_{43} x_{1}\right) \\
& +\left(\frac{\partial f}{\partial y_{3}}(\mathbf{x}) \frac{\partial g}{\partial y_{2}}(\mathbf{x})-\frac{\partial f}{\partial y_{2}}(\mathbf{x}) \frac{\partial g}{\partial y_{3}}(\mathbf{x})\right)\left(s_{42} y_{3}-s_{43} y_{2}-s_{44} x_{1}\right) \\
& +\left(\frac{\partial f}{\partial x_{1}}(\mathbf{x}) \frac{\partial g}{\partial y_{2}}(\mathbf{x})-\frac{\partial f}{\partial y_{2}}(\mathbf{x}) \frac{\partial g}{\partial x_{1}}(\mathbf{x})\right)\left(s_{22} y_{3}-s_{32} y_{2}-s_{42} x_{1}\right)
\end{aligned}
$$

where $\mathbf{x} \in \mathcal{L}_{+}(4)$ is a upper triangular matrix given by Equation (32).

We recognize in (40) the Lie-Poisson structure related to $\mathfrak{g}_{4,1}$ if we take $S_{3}$, as in Table 8 , and put $x_{1}=y_{2}=0$. An isomorphism is given by the mapping $\left(x_{2}, y_{1}, x_{3}, y_{3}\right) \mapsto$ $\left(e_{1}, e_{2}, e_{3}, e_{4}\right)$. If we take the isomorphism $\left(-x_{2}, x_{1}, y_{1}, y_{2}\right) \mapsto\left(e_{1}, e_{2}, e_{3}, e_{4}\right)$ and as nonzero element in matrix $S_{3}$, we put $s_{42}=-1$ and $x_{3}=y_{3}=0$, then the bracket (40) gives us the algebra $\mathfrak{g}_{4,8}$. We obtain the Lie algebra $\mathfrak{g}_{4,10}$ by taking matrix $S_{3}$ as in Table 8 . In this case an isomorphism is given by $\left(e_{1}, e_{2}, e_{3}, e_{4}\right) \mapsto\left(2 x_{3}, x_{1}+y_{1}, x_{2}+y_{2}, y_{3}\right)$. We put $x_{1}=y_{1}$, $x_{2}=y_{2}, x_{3}=2 \tilde{x}_{3}$. Let us also note that using Lie bundles gives matrix representations of these Lie algebras as subsets of $M_{6}(\mathbb{R})$. Generators of these subsets are presented in the third column of Table 8 .

Lie algebras $\mathfrak{g}_{4,2}^{a}, \mathfrak{g}_{4,5}^{a b}, \mathfrak{g}_{4,6}^{a b}$ were obtained in the construction called the second bundle (see Table 7). If so, we have also their representation for sub-cases when $a=2, a=1-b$, $a=2 b$, respectively, see Table 7 .

The three remaining Lie algebras, i.e., $\mathfrak{g}_{4,7}, \mathfrak{g}_{4,9}^{b}, \mathfrak{g}_{4,11}^{a}$, can be obtained using the biHamiltonian construction on the Lie algebras from Tables 7 and 8 . Lie algebra $\mathfrak{g}_{4,11}$ can be obtained from algebras $\mathfrak{g}_{4,10}$ and $\mathfrak{g}_{4,5}^{\frac{1}{2} \frac{1}{2}}$. We have $\mathfrak{g}_{4,11}=\mathfrak{g}_{4,10}+2 a \mathfrak{g}_{4,5}^{\frac{1}{2} 2}$. In a similar way, we can get $\mathfrak{g}_{4,9}^{b}$ from $\mathfrak{g}_{4,8}$ and $\mathfrak{g}_{4,5}^{01}: \mathfrak{g}_{4,9}^{b}=\mathfrak{g}_{4,8}+(1+b) \mathfrak{g}_{4,5}^{01}$. The last Lie algebra $\mathfrak{g}_{4,7}$ is given by taking Lie algebras $\mathfrak{g}_{4,10}, \mathfrak{g}_{4,2}^{2}, \mathfrak{g}_{4,6}^{00}: \mathfrak{g}_{4,7}=\mathfrak{g}_{4,10}+\mathfrak{g}_{4,2}^{2}-\mathfrak{g}_{4,6}^{00}$. 
Table 8. The third Lie bundle (first part).

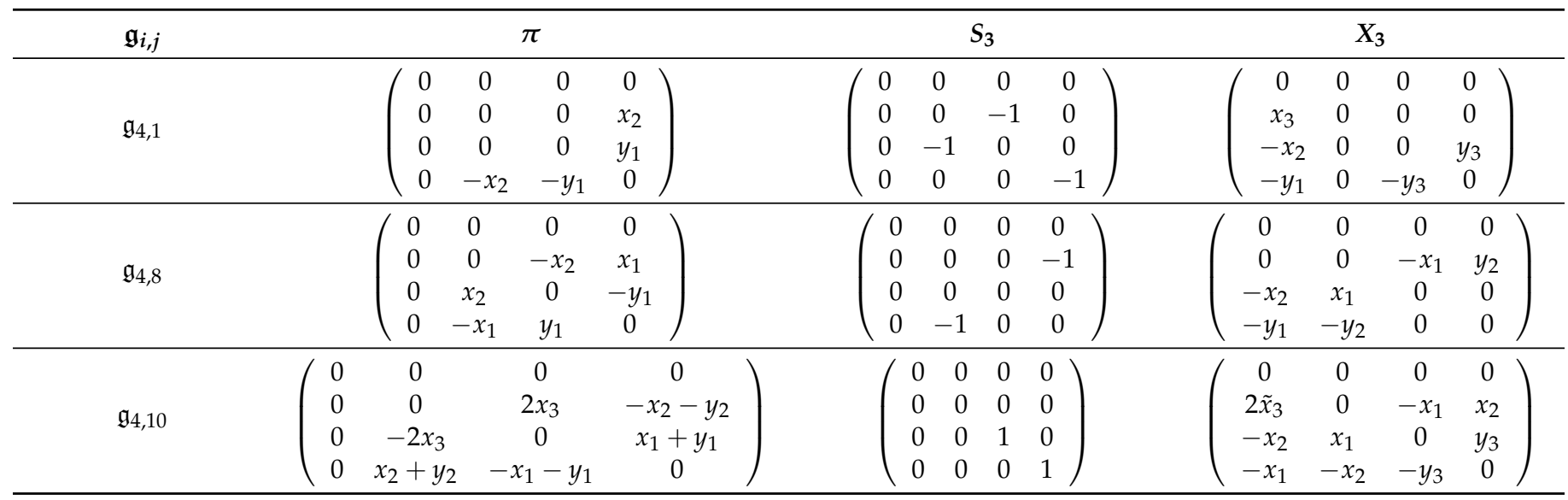

\section{Conclusions}

We proposed a new approach to the classification of low-dimensional Lie algebras using concepts well known in the theory of integrable systems, such as the bi-Hamiltonian structure. In the case of three-Dimensional Lie algebras, we were able to describe them using two families of linear bundles of Lie algebras. In the case of the four-dimensional Lie algebras, we described them in terms of three such families. In the future, we plan to use these tools to study higher dimensional Lie algebras.

The bi-Hamilton property is very useful in the study of integrable systems. Let us consider the well-known Euler equations for $n=3$ :

$$
\begin{aligned}
& \frac{d x_{1}}{d t}=\left(s_{22}-s_{33}\right) x_{2} x_{3}, \\
& \frac{d x_{2}}{d t}=\left(s_{33}-s_{11}\right) x_{1} x_{3}, \\
& \frac{d x_{3}}{d t}=\left(s_{11}-s_{22}\right) x_{1} x_{2} .
\end{aligned}
$$

The Euler top describes the rotation of a heavy rigid body around its fixed center of mass without any forces acting on the body. Equation (41) possess a bi-Hamiltonian structure

$$
\frac{d x_{i}}{d t}=\left\{H_{1}, x_{i}\right\}_{1}=\left\{H_{2}, x_{i}\right\}_{2}, \quad i=1,2,3 .
$$

The Poisson brackets $\{\cdot, \cdot\}_{1}$ and $\{\cdot, \cdot\}_{2}$ are defined by Equation (21), where we restrict our considerations to diagonal matrices $S_{1}=\mathbf{1}$ and $S_{1}=\operatorname{diag}\left(s_{11}, s_{22}, s_{33}\right)$. The Casimirs for these structures assume the following form (see Equation (27))

$$
C_{1}=\frac{1}{2}\left(x_{1}^{2}+x_{2}^{2}+x_{3}^{2}\right), \quad C_{2}=-\frac{1}{2}\left(s_{11} x_{1}^{2}+s_{22} x_{2}^{2}+s_{33} x_{3}^{2}\right),
$$

respectively. Choosing as the Hamiltonian $H_{1}$ the Casimir $\mathrm{C}_{2}$ and as Hamiltonian $\mathrm{H}_{2}$ the Casimir $C_{1}$, we obtain Equation (42). Of course, the first Poisson bracket is associated with the Lie algebra $\mathfrak{s o}(3)$. We can look at the second Poisson bracket as a collection of three brackets corresponding to the Lie algebra $\mathfrak{g}_{3,1}$ for matrices:

$$
S_{1}=\left(\begin{array}{lll}
1 & 0 & 0 \\
0 & 0 & 0 \\
0 & 0 & 0
\end{array}\right), \quad S_{1}=\left(\begin{array}{lll}
0 & 0 & 0 \\
0 & 1 & 0 \\
0 & 0 & 0
\end{array}\right), \quad S_{1}=\left(\begin{array}{lll}
0 & 0 & 0 \\
0 & 0 & 0 \\
0 & 0 & 1
\end{array}\right),
$$

respectively (see Table 1). 
Now, we discuss another example of a Lie bundle. We now turn to the Clebsch system of the motion of a rigid body in an ideal fluid

$$
\begin{aligned}
\frac{d x_{1}}{d t} & =\left(s_{22}-s_{33}\right) x_{2} x_{3}, \\
\frac{d x_{2}}{d t} & =x_{1} x_{3}-y_{1} y_{3}, \\
\frac{d x_{3}}{d t} & =y_{1} y_{2}-x_{1} x_{2}, \\
\frac{d y_{1}}{d t} & =x_{2} y_{3}-x_{3} y_{2}, \\
\frac{d y_{2}}{d t} & =\left(s_{44}-s_{22}\right) x_{3} y_{1}, \\
\frac{d y_{3}}{d t} & =\left(s_{33}-s_{44}\right) x_{2} y_{1} .
\end{aligned}
$$

In vector notation, the system hast the form:

$$
\begin{aligned}
& \frac{d \vec{x}}{d t}=\vec{x} \times \vec{y}, \\
& \frac{d \vec{y}}{d t}=\vec{x} \times(\tilde{S} \vec{x}),
\end{aligned}
$$

where $\vec{x}=\left(x_{3}, x_{2}, y_{1}\right), \vec{y}=\left(-y_{3},-y_{2},-x_{1}\right)$ and $\tilde{s}=\left(\begin{array}{ccc}s_{22} & 0 & 0 \\ 0 & s_{33} & 0 \\ 0 & 0 & s_{44}\end{array}\right)$. Equation (45) possesses a bi-Hamiltonian structure

$$
\frac{d x_{i}}{d t}=\left\{H_{1}, x_{i}\right\}_{1}=\left\{H_{2}, x_{i}\right\}_{2}, \quad \frac{d y_{i}}{d t}=\left\{H_{1}, y_{i}\right\}_{1}=\left\{H_{2}, y_{i}\right\}_{2}, \quad i=1,2,3 .
$$

The Poisson bracket $\{\cdot, \cdot\}_{1}$ is defined by Equation (40), where we restrict our considerations to diagonal matrix $S_{3}=\operatorname{diag}(0,1,1,1)$. However, the same Poisson bracket is also obtained from Equation (12), where $X_{3}, Y_{3} \in \mathcal{A}_{1,1,1}(4)$. The second Poisson bracket $\{\cdot, \cdot\}_{2}$ is given by Equation (12), where $S=\operatorname{diag}\left(-1, s_{22}, s_{33}, s_{44}\right)$. We consider the Poisson pencil $\{\cdot, \cdot\}_{\lambda}=\{\cdot, \cdot\}_{1}-\lambda\{\cdot, \cdot\}_{2}, \lambda \in \mathbb{R}$. This Poisson bracket can also be obtained from Equation (12) for matrix $S_{\lambda}=\operatorname{diag}\left(\lambda, 1-\lambda s_{22}, 1-\lambda s_{33}, 1-\lambda s_{44}\right)$. The Casimir (one of the functions) for this structure assumes the following form (see Equation (15)):

$$
\begin{gathered}
C_{1}^{\lambda}=\frac{1}{2\left(1-\lambda s_{22}\right)} x_{3}^{2}+\frac{1}{2\left(1-\lambda s_{33}\right)} x_{2}^{2}+\frac{1}{2\left(1-\lambda s_{44}\right)} y_{1}^{2} \\
+\frac{\lambda}{2\left(1-\lambda s_{33}\right)\left(1-\lambda s_{44}\right)} y_{3}^{2}+\frac{\lambda}{2\left(1-\lambda s_{22}\right)\left(1-\lambda s_{44}\right)} y_{2}^{2}+\frac{\lambda}{2\left(1-\lambda s_{22}\right)\left(1-\lambda s_{33}\right)} x_{1}^{2} .
\end{gathered}
$$

This Casimir function can be expanded into a power series with respect to $\lambda$. By using power series decomposition, we get $C_{1}^{\lambda}=C_{1}+\lambda H_{1}+\ldots$, where

$$
C_{1}=\frac{1}{2}\left(x_{3}^{2}+x_{2}^{2}+y_{1}^{2}\right)
$$

is the Casimir for the first bracket and

$$
H_{1}=\frac{1}{2}\left(s_{22} x_{3}^{2}+s_{33} x_{2}^{2}+s_{44} y_{1}^{2}+y_{3}^{2}+y_{2}^{2}+x_{1}^{2}\right) .
$$

Choosing as the first Hamiltonian the function $\mathrm{H}_{1}$ and as Hamiltonian $\mathrm{H}_{2}$ the Casimir $C_{1}$, we obtain Equation (48). The first Poisson bracket is associated with the Lie algebra $\mathfrak{e}(3)$. 
We can look at the second Poisson bracket as a collection of four brackets corresponding to the nilpotent Lie algebra $\mathfrak{g}_{6,3}$ (we use the classification from [30]) for matrices:

$$
\begin{aligned}
& S=\left(\begin{array}{llll}
1 & 0 & 0 & 0 \\
0 & 0 & 0 & 0 \\
0 & 0 & 0 & 0 \\
0 & 0 & 0 & 0
\end{array}\right), \quad S=\left(\begin{array}{lllll}
0 & 0 & 0 & 0 \\
0 & 1 & 0 & 0 \\
0 & 0 & 0 & 0 \\
0 & 0 & 0 & 0
\end{array}\right), \\
& S=\left(\begin{array}{llll}
0 & 0 & 0 & 0 \\
0 & 0 & 0 & 0 \\
0 & 0 & 1 & 0 \\
0 & 0 & 0 & 0
\end{array}\right), \quad S=\left(\begin{array}{llll}
0 & 0 & 0 & 0 \\
0 & 0 & 0 & 0 \\
0 & 0 & 0 & 0 \\
0 & 0 & 0 & 1
\end{array}\right),
\end{aligned}
$$

respectively.

Author Contributions: Conceptualization, A.D. and K.W.; formal analysis, A.D. and K.W. All authors have read and agreed to the published version of the manuscript.

Funding: This article has received financial support from the Polish Ministry of Science and Higher Education under subsidy for maintaining the research potential of the Faculty of Mathematics, University of Bialystok.

Institutional Review Board Statement: Not applicable.

Informed Consent Statement: Not applicable.

Data Availability Statement: No data and materials were used to support this study.

Conflicts of Interest: The authors declare that they have no competing interests.

\section{References}

1. Bolsinov, A.V.; Borisov, A.V. Compatible Poisson brackets on Lie algebras. Math. Notes 2002, 72, 10-30. [CrossRef]

2. Trofimov, V.V.; Fomenko, A.T. Algebra and Geometry of Integrable Hamiltonian Differential Equations; Factorial: Moscow, Russia, 1995. (In Russian)

3. Yanovski, A.B. Linear bundles of Lie algebras and their applications. J. Math. Phys. 2000, 41, 7869. [CrossRef]

4. Morosi, C.; Pizzocchero, L. On the Euler Equation: Bi-Hamiltonian Structure and Integrals in Involution. Lett. Math. Phys. 1996, 37, 117-135. [CrossRef]

5. Dobrogowska, A.; Ratiu, T.S. Integrable Systems of Neumann Type. J. Dyn. Differ. Equ. 2015, 27, 533-553. [CrossRef]

6. Odzijewicz, A.; Dobrogowska, A. Integrable Hamiltonian systems related to the Hilbert-Schmidt ideal. J. Geom. Phys. 2011, 61, 1426-1445. [CrossRef]

7. Yanovski, A.B. Bundles of Lie algebras and compatible Poisson brackets. Int. J. Mod. Phys. A 2002, 17, 946-950. [CrossRef]

8. Bloch, A.M.; Brînzănescu, V.; Iserles, A.; Marsden, J.E.; Ratiu, T.S. A class of integrable flows on the space of symmetric matrices. Commun. Math. Phys. 2009, 290, 399-435. [CrossRef]

9. Cantor, I.L.; Persits, D.E. About closed bundles of linear Poisson brackets. In Proceedings of the IX USSR Conference in Geometry Proceedings, Kishinev, Moldova, 12-16 December 1988.

10. Dali, B. Bundles of Lie algebras. Int. J. Geom. Methods Mod. Phys. 2013, 10, 1350045. [CrossRef]

11. Kiranagi, B.S.; Madhu, B.; Ajaykumar, K. On smooth Lie algebra Bundles. Int. J. Algebra 2017, 11, 247-254. [CrossRef]

12. Nijenhuis, A.; Richardson, R. Deformations of Lie algebra structures. J. Math. Mech. 1967, 17, 89-105. [CrossRef]

13. Inönü, E.; Wigner, E.P. On the contraction of groups and their representations. Proc. Natl. Acad. Sci. USA 1953, 39, 510-524. [CrossRef] [PubMed]

14. Inönü, E.; Wigner, E.P. On the particular type of convergence to a singular matrix. Proc. Natl. Acad. Sci. USA 1953, 40, 119-121. [CrossRef] [PubMed]

15. Santander, M.; Herranz, F.J. Geometries of Orthogonal Groups and their Contractions: A Unified Classical Deformation Viewpoint. Int. J. Mod. Phys. A 1997, 12, 99-107. [CrossRef]

16. Santander, M.; Herranz, F.J. Casimir invariants for the complete family of quasisimple orthogonal algebras. J. Phys. A Math. Gen. 1997, 30, 5411-5426.

17. Dobrogowska, A.; Odzijewicz, A. Integrable Systems related to deformed so(5). SIGMA Symmetry Integr. Geom. Methods Appl. 2014, 10, 1-18. [CrossRef]

18. Ballesteros, A.; Gubitosi, G.; Gutierrez-Sagredo, I.; Herranz, F.J. The $\kappa$-Newtonian and $\kappa$-Carrollian algebras and their noncommutative spacetimes. Phys. Lett. B 2020, 805, 135461. [CrossRef] 
19. Dobrogowska, A.; Odzijewicz, A. Integrable relativistic systems given by Hamiltonians with momentum-spin-orbit coupling. Regul. Chaotic Dyn. 2012, 17, 492-505. [CrossRef]

20. Magri, F. A simple model of integrable Hamiltonian equation. J. Math. Phys. 1978, 19, 1156-1162. [CrossRef]

21. Bianchi, L. Lezioni sulla teoria dei gruppi continui finiti di transformazioni; Enrico Spoerri: Pisa, Italy, 1918.

22. Mubarakzyanov, G.M. On solvable Lie algebras. Izv. Vys. Ucheb. Zaved. Mat. 1963, 1, 114-123.

23. MacCallum, M.A.H. On the classification of the real four-dimensional Lie algebras. In On Einstein's Path; Springer: Berlin, Germany, 1999; pp. 299-317.

24. Boza, L.; Fedriani, E.M.; Nunez, J.; Tenorio, A.F. A Historical review of the classifications of Lie algebras. Rev. Union Mat. Argent. 2013, 54, 75-99.

25. de Graaf, W.A. Classification of Solvable Lie Algebras. Exp. Math. 2005, 14, 15-25. [CrossRef]

26. Popovych, R.O.; Boyko, V.M.; Nesterenko, M.O.; Lutfullin, M.W. Realizations of real low-dimensional Lie algebras. J. Phys. A Math. Gen. 2003, 36, 7337. [CrossRef]

27. Šnobl, L.; Winternitz, P. Classification and Identification of Lie Algebras. In CRM Monograph Series; American Mathematical Society: Providence, RI, USA, 2014.

28. Dobrogowska, A. R-matrix, Lax pair, and multiparameter decompositions of Lie algebras. J. Math. Phys. 2015, 56, 1-11. [CrossRef]

29. Dobrogowska, A.; Goliński, T. Lie bundle on the space of deformed skew-symmetric matrices. J. Math. Phys. 2014, 55, 1-14. [CrossRef]

30. Patera, J.; Sharp, R.T.; Winternitz, P.; Zassenhaus, H. Invariants of real low dimension Lie algebras. J. Math. Phys. 1976, 17, 986-994. [CrossRef]

31. Dali, B. Linear family of Lie brackets on the space of matrices Mat $(n \times m)(K)$ and Ado's Theorem. arXiv 2008, arXiv:0807.1851.

32. Ghanam, R.A.; Strugar, I.; Thompson, G. Matrix representations for low dimensional Lie algebras. Extr. Math. 2005, 20, 151-184. 